\title{
Strümpfe als Standbein der Thromboseprophylaxe
}

\section{Hier steht eine Anzeige.}

\author{
Medizinische Kompressionsstrümpfe senken das Risiko für venöse Thrombo- \\ embolien. Auch in der Ära moderner Antikoagulanzien haben sie noch nicht \\ ausgedient. In den Leitlinien nehmen Sie - allein oder in Kombination mit \\ anderen Prophylaxemaßnahmen - nach wie vor einen festen Platz ein.
}

- Für chirurgische Patienten ist der Nutzen von medizinischen Thromboseprophylaxe-Strümpfen durch ein Cochrane-Review belegt (Sachdeva A et al., 2010). Strumpfträger ohne weitere Prophylaxe entwickeln demnach seltener tiefe Venenthrombosen (TVT) als Patienten, bei denen gar keine Prophylaxe betrieben wird (13\% vs. 26\%). Die Cochrane-Wissenschaftler leiten daraus eine Empfehlung für alle Patienten mit erhöhtem TVT-Risiko ab: Sofern keine Kontraindikationen bestehen, sollte die Verordnung von Kompressionsstrümpfen erwogen werden.

Werden die Strümpfe mit einer anderen Prophylaxemaßnahme kombiniert, scheint dies der Cochrane-Analyse zufolge TVT wirksamer zu verhindern als die jeweilige Hintergrundtherapie allein (4\% vs. 16\%). In der S3-Leitlinie zur Prophylaxe der venösen Thromboembolie (VTE) heißt es: „Eine eindeutige Überlegenheit der Kombination aus physikalischen und medikamentösen Maßnahmen gegenüber einer alleinigen medikamentösen Prophylaxe wurde bisher nicht allgemein zweifelsfrei, sondern nur für einzelne Indikationsbereiche belegt.“

\section{Prophylaxe je nach Risiko}

Laut Leitlinie können physikalische Maßnahmen, insbesondere Kompressionsstrümpfe, zusätzlich zu einer indizierten Prophylaxe eingesetzt werden. Diese besteht bei Patienten mit niedrigem Risiko aus Basismaßnahmen wie früher Mobilisierung und bei Patienten mit mittlerem bis hohem Risiko aus $\mathrm{Ba}$ -
Voraussetzung für den Erfolg: Der Strumpf muss sitzen.

sismaßnahmen plus Antikoagulation.

Ein niedriges Risiko besteht $z$. B. bei kleinen operativen Eingriffen, ein mittleres bei gelenkübergreifender Immobilisation der unteren Extremität oder bei stationär behandlungsbedürftiger maligner Erkrankung, und ein hohes u.a. bei größeren Eingriffe an Wirbelsäule, Becken, Hüft- und

Kniegelenk oder Schlaganfall mit Beinparese. Überdies empfehlen die Leitlinienautoren das Anlegen von Kompressionsstrümpfen, wenn eine angezeigte medikamentöse Prophylaxe wegen Kontraindikationen nicht möglich ist, z.B bei stationären Krebspatienten mit hohem Blutungsrisiko.

Die Schutzwirkung der Kompressionsstrümpfe beruht vermutlich auf einer Erhöhung der Blutströmungsgeschwindigkeit, die der Bildung von Thromben vorbeugen kann. Voraussetzung dafür ist, dass die Thromboseprophylaxe „sitzt“: Der lokale Anpressdruck der Strümpfe soll in der Fesselregion ca. 18 mmHg betragen und nach proximal kontinuierlich abnehmen, Einschnürungen sind unbedingt zu vermeiden.

Prinzipiell kontraindiziert sind Kompressionsstrümpfe bei kritischen peripheren arteriellen Durchblutungsstörungen, schweren Neuropathien, ausgeprägten peripheren Ödemen und lokalen Infekten, Nekrosen und Verletzungen. Dr. Beate Schumacher . 